\title{
含苯并环丁烯基团的热固性聚碳酸酯的合成及性能研究
}

\author{
李智勇 $a, b$ 曹卫国*,a 孙晶 $*, b$ 房强 $*, b$ \\ $\left({ }^{a}\right.$ 上海大学化学系 上海 200444) \\ $\left({ }^{b}\right.$ 中国科学院上海有机化学研究所 上海 200032)
}

\begin{abstract}
摘要 报道了一种新型的含有苯并环丁烯基团的热固性芳香族聚碳酸酯的合成方法. 该聚合物具有良好的溶解性，可 用溶液法进行加工. 该聚合物在高温固化后表现出优异的热稳定性，在氮气中 $5 \%$ 的热重损失温度高达 $451{ }^{\circ} \mathrm{C}$, 在 $1000{ }^{\circ} \mathrm{C}$ 下的残炭率为 $33 \%$. 该聚合物还具有良好疏水性，在沸水中浸泡 $24 \mathrm{~h}$ 后的吸水率低至 $0.21 \%$. 为了进一步研究 苯并环丁烯基团的引入对芳香族聚碳酸酯性能的影响, 利用双酚 A 单体合成了部分侧链带有苯并环丁烯基团的共聚物 以及线性双酚 $\mathrm{A}$ 型聚碳酸酯. 通过性能对比研究可知, 苯并环丁烯基团的引入能有效提高芳香族聚碳酸酯的耐热性和 疏水性.
\end{abstract}

关键词聚碳酸酯; 苯并环丁烯; 合成; 热固性聚合物

\section{Synthesis and Properties of Benzocyclobutene-Functionalized Thermosetting Polycarbonate}

\author{
Li, Zhiyong ${ }^{a, b}$ Cao, Weiguo ${ }^{*, a} \quad$ Sun, Jing ${ }^{*, b} \quad$ Fang, Qiang ${ }^{*, b}$ \\ ( ${ }^{a}$ Department of Chemistry, Shanghai University, Shanghai 200444) \\ ( ${ }^{b}$ Shanghai Institute of Organic Chemistry, Chinese Academy of Sciences, Shanghai 200032)
}

\begin{abstract}
A novel benzocyclobutene (BCB)-functionalized polycarbonate was synthesized. This polymer showed good solubility in organic solvents and could be processed by solution method. After cured at high temperature, the polymer exhibited high thermostability with $5 \%$ weight loss temperature of $451{ }^{\circ} \mathrm{C}$ and a char yield of $33 \%$ at $1000{ }^{\circ} \mathrm{C}$ in nitrogen. The cured polymer showed good hydrophobicity with water uptake of $0.21 \%$ after immersion in boiling water for $24 \mathrm{~h}$. Moreover, a copolymer and poly(bisphenol A carbonate) were synthesized by bisphenol A monomer to study the influence of the existence of $\mathrm{BCB}$ groups on the properties of the aromatic polycarbonate. The results of comparative study showed that BCB groups can effectively improve the thermostability and hydrophoblicity of aromatic polycarbonates.
\end{abstract}

Keywords polycarbonate; benzocyclobutene; synthesis; thermosetting polymer

芳香族聚碳酸酯是一种常用的工程塑料, 它具有优 良的抗冲击性、较高的拉伸强度、良好的耐热性能、介 电性能和透光性能, 因而广泛应用于电子电器、汽车、 机械、光盘和航空航天等各个方面 ${ }^{[1-6]}$, 具有十分重要 的研究意义和经济价值. 芳香族聚碳酸酯的主要缺点是 熔融粘度大, 成型加工较困难, 从而限制了其应用范围. 因此通过对其化学结构改性以改善其加工性的研究工 作受到越来越多的关注. 对芳香族聚碳酸酯结构改性的 方法有多种, 例如发展含有支化结构的聚碳酸酯并且将 其与线性聚碳酸酯共混, 可以有效地降低其熔融粘
度 ${ }^{[7,8]}$, 但是支化结构的引入会使聚碳酸酯的耐热性有 所降低. 还有一种常用的方法是在聚碳酸酯的主链中引 入如葱、芴等具有较大体积的刚性结构单元 ${ }^{[0 \sim 12]}$, 大体 积基团可以降低聚合物分子链间的作用以改善其加工 性, 并且刚性结构基团有利于提高聚碳酸酯的热稳定 性. 利用该方法改性的聚碳酸酯在有机溶剂中具有较好 的溶解性, 其玻璃化转变温度可达到 $230{ }^{\circ} \mathrm{C}$ 左右. 虽然 这类芳香族聚碳酸酯具有较高的热稳定性, 但仍然无法 满足在更高温度条件下使用的要求. 因此如何开发具有 良好加工性及高耐热性的聚碳酸酯仍需进一步探索. 通

* E-mail: qiangfang@sioc.ac.cn; sunjing@sioc.ac.cn; wgcao@staff.shu.edu.cn Received April 7, 2016; revised May 30, 2016; published online June 2, 2016. P Project supported by the Natural Science Foundation of China (NSFC, No. 21374131, 21574146 and 21504103) and the Science and Technology Commission of Shanghai Municipality (15ZR1449200).

国家自然科学基金基金(No. 21374131, 21574146, 21504103), 上海市自然科学基金(No. 15ZR1449200)资助项目. 
常而言, 提高分子量是提高聚合物耐热性的一种常用手 段, 然而随着分子量的提升, 聚合物的溶解性、可加工 性等方面则会相应地下降. 综合来看, 在不影响聚合物 溶解性及可加工性的前提下, 引入可固化基团, 通过固 化交联, 使聚合物形成网状结构, 是提高材料性能的一 种有效方法. 因此, 我们尝试发展一类带有可固化基团 的聚碳酸酯, 期望在改善其加工性的同时, 使其获得优 异的热稳定性.

在能引入聚碳酸酯的可固化基团中, 热固性基团是 比较好的选择. 因为引入热固性基团, 不用加交联剂, 只需加热就可使聚合物交联, 方法简单. 不同的热固性 基团还可以改善聚合物其他的性能, 如绝缘性能、阻燃 性能等, 能使其应用到更广阔的领域中. 在热固性基团 中, 苯并环丁烯是一种具有高反应活性的热固性基团, 其含有四元环结构, 在加热条件下开环发生 Diels-Alder

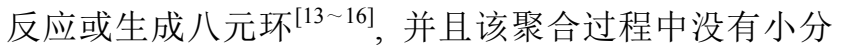
子放出, 因而苯并环丁烯常用作封端基团或交联基团开 发为树脂 ${ }^{[17 ~ 22]}$. 含苯并环丁烯(BCB)的树脂均展现了较 高的耐热性、较低的介电常数和良好的力学性能等. 鉴 于苯并环丁烯基团的优异性能, 我们设计合成了含有苯 并环丁烯的双酚单体 BCB-DP, (结构如图 1 所示), 并将 其与三光气反应制得聚碳酸酯 BPC. 由于该聚碳酸酯侧 链含有苯并环丁烯基团, 使其具有良好的溶解性, 可用 溶液加工的方式制成薄膜, 加工性能良好. 该聚合物可 加热固化得到立体交联网状结构的聚碳酸酯(PBPC), 其在氮气中 $5 \%$ 的热重损失温度高达 $451{ }^{\circ} \mathrm{C}$, 在 $1000{ }^{\circ} \mathrm{C}$ 下的残炭率为 $33 \%$, 展现了优异的热稳定性. 为了进一步研究苯并环丁烯基团的引入对聚碳酸酯性 能的影响, 我们利用 BCB-DP、双酚 A 单体和三光气分 别制得了部分侧链带有含苯并环丁烯基团的共聚物 (BA-BPC) 以及线性双酚 A 型聚碳酸酯(BAPC), 并将三 种聚合物的性能进行了比较研究. 对比结果显示, 苯并 环丁烯基团的引入可使聚碳酸酯热固化后, 获得更高的 热稳定性，并且其疏水性也有所提高.

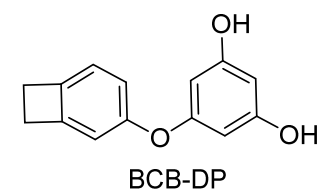

图 1 含苯并环丁烯基团的单体 BCB-DP 的化学结构

Figure 1 Chemical structure of monomer BCB-DP

\section{1 结果与讨论}

\section{1 聚碳酸酯单体的合成与表征}

单体 BCB-DP 的合成路线如 Scheme 1 所示, 首先采 用均三甲氧基苯为原料在正十二烷基硫醇作用下脱去
一个甲氧基制得化合物 $\mathbf{1}^{[23]}$. 化合物 $\mathbf{1}$ 在 $N$-甲基吡咯烷 酮(NMP)中与氢氧化钾反应，利用甲苯回流除水可得到 化合物 1 的酚钾盐. 该酚钾盐在碘化亚铜催化下与 4-溴 苯并环丁烯反应，可得到 3,5-二甲氧基苯基-4-苯并环丁 烯基醚(2)，最后利用碘化铝脱去甲氧基便可制得含苯 并环丁烯的间苯二酚单体 BCB-DP.
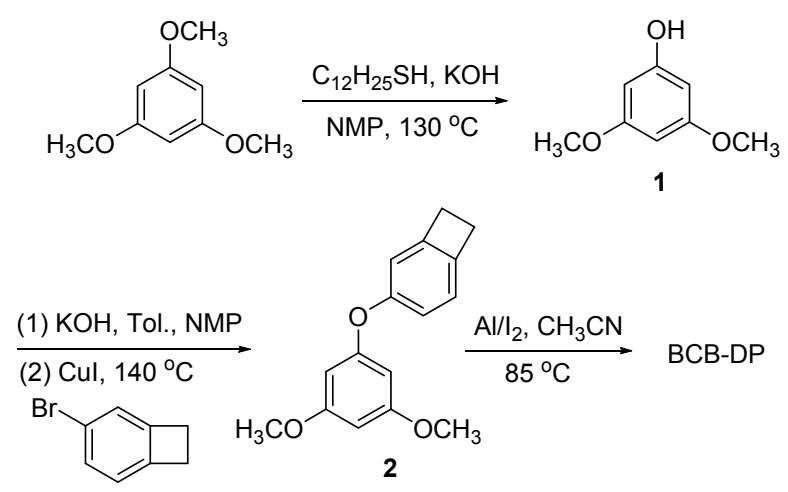

图式 1 聚碳酸酯单体 BCB-DP 的合成路径

Scheme 1 Synthesis of the polycarbonate monomer BCB-DP

通过 ${ }^{1} \mathrm{H}$ NMR, FT-IR 和高分辨质谱等表征手段对单 体 BCB-DP 的化学结构进行了表征. 图 2 为单体 BCB-DP 的核磁氢谱. 化学位移在 $\delta 6.7 \sim 7.1$ 和 $5.9 \sim 6.1$ 处的信号峰分别归属于苯并环丁烯上的苯环和含酚羟 基苯环上的质子峰， $\delta 5.0 \sim 5.2$ 处为羟基的质子峰 $\delta$ $3.0 \sim 3.2$ 处为苯并环丁烯上两个亚甲基上的质子峰. 图 3 为单体 BCB-DP 的红外图谱, 其中 $1468 \mathrm{~cm}^{-1}$ 为苯并 环丁烯亚甲基上 $\mathrm{C}-\mathrm{H}$ 键平面拉伸所产生的特征峰 ${ }^{[25]}$. 以上结果表明, 所获得产物的化学结构与目标产物相符 合.

\section{2 聚碳酸酯的合成}

在成功获得单体 BCB-DP 后，我们将其与三光气反 应制备侧链带有苯并环丁烯基团的聚碳酸酯(BPC). 聚 合物的合成如 Eq. 1 所示, 以单体 BCB-DP 和三光气 (BTC) 为原料, 三乙胺作为缚酸剂, 二氯甲烷为溶剂, 在室温条件下反应 $5 \mathrm{~h}$ 后加入封端剂甲醇, 经过后处理 后, 最终可得到白色固体. 通过 ${ }^{1} \mathrm{H}$ NMR、FT-IR 和 GPC 对所得聚合物进行了表征. 图 4 和图 5 分别为聚合物 $\mathrm{BPC}$ 的核磁氢谱和红外谱图. 在图 4 中 $\delta 3.0 \sim 3.2$ 处的 信号峰为苯并环丁烯亚甲基的质子峰. 图 5 中 1468 $\mathrm{cm}^{-1}$ 的吸收峰同样证实了苯并环丁烯基团的存在. 红 外谱图中在 $1780 \mathrm{~cm}^{-1}$ 处有明显的羰基吸收峰. 以上结 果确定了聚合物 BPC 的化学结构. 通过 GPC 测得其数 均分子量为 25000 , 分散度为 1.6. 该聚合物可以良好地 溶解在 THF、氯仿、二氯甲烷等常用有机溶剂中, 并且 其聚合物溶液可以用旋涂或滴涂的方法制备成聚合物 薄膜. 


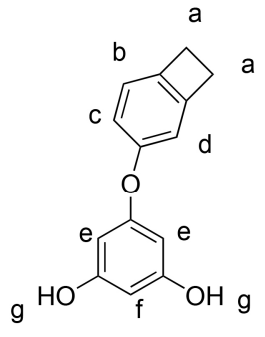

e

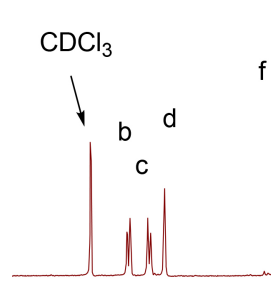

$7.5 \quad 7.0$

图 2 单体 BCB-DP 的核磁氢谱谱图

Figure $2{ }^{1} \mathrm{HNMR}$ spectrum of $\mathrm{BCB}-\mathrm{DP}\left(\mathrm{CDCl}_{3}, 400 \mathrm{MHz}\right)$

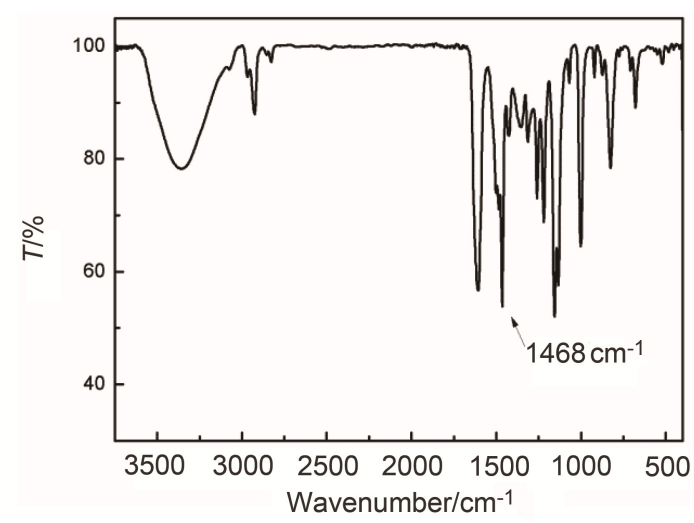

图 3 单体 BCB-DP 的红外图谱

Figure 3 FT-IR spectrum of BCB-DP<smiles>Oc1cc(O)cc(Oc2ccc3c(c2)CC3)c1</smiles><smiles>CC(C)(c1ccc(O)cc1)c1ccc(O)cc1</smiles>

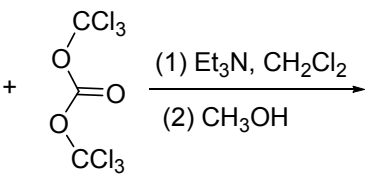<smiles>COC(=O)Oc1ccc(C(C)(C)c2ccc(OC(=O)OCc3cc(OC(=O)OC)cc(Oc4ccc5c(c4)CC5)c3)cc2)cc1</smiles>

BPC: $m=0, M_{\mathrm{n}}=25000, M_{\mathrm{w}} / M_{\mathrm{n}}=1.6$;

BA-BPC: $m / n=1.5, M_{\mathrm{n}}=20500, M_{\mathrm{w}} / M_{\mathrm{n}}=1.7$;

BAPC: $n=0, M_{\mathrm{n}}=19400, M_{\mathrm{w}} / M_{\mathrm{n}}=1.7$

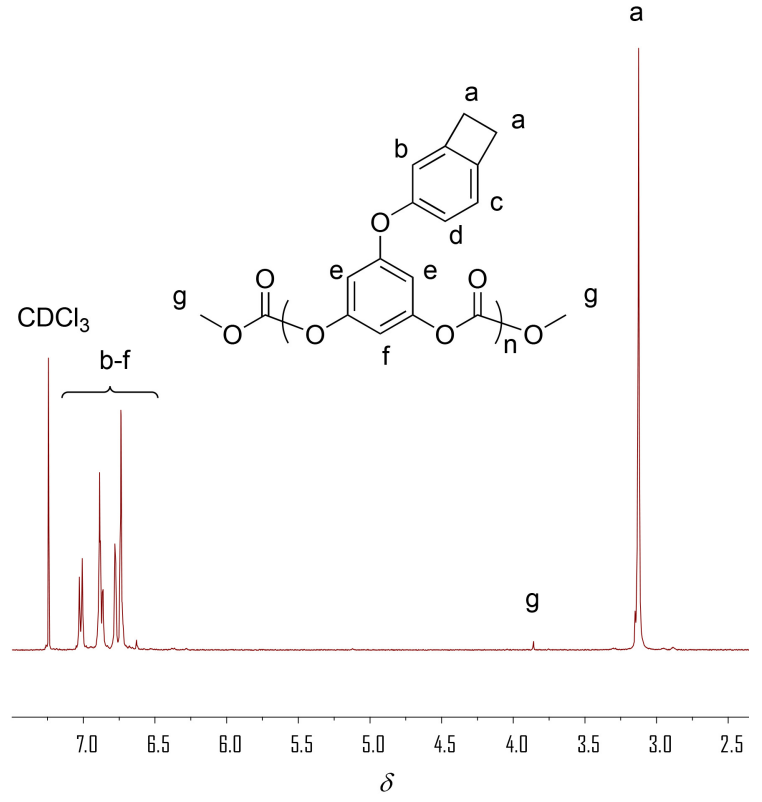

图 4 聚合物 $\mathrm{BPC}$ 的核磁氢谱谱图

Figure $4{ }^{1} \mathrm{H}$ NMR spectrum of $\mathrm{BPC}\left(\mathrm{CDCl}_{3}, 400 \mathrm{MHz}\right)$

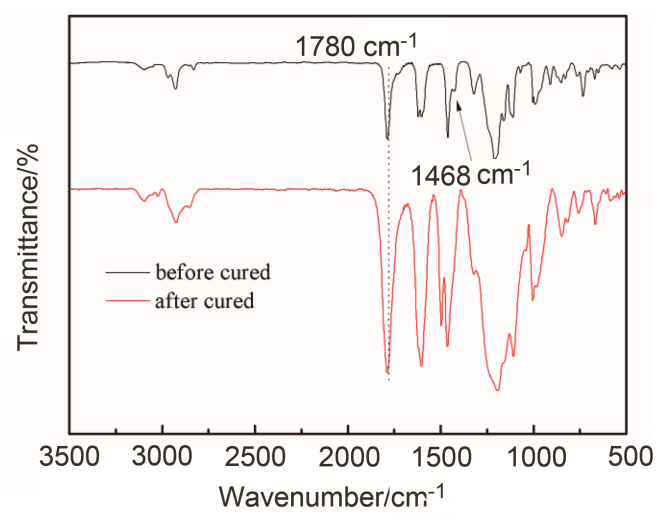

图 5 聚碳酸酯 BPC 固化前后的红外谱图

Figure5 FT-IR spectrum of BPC before and after cured

为了进一步研究苯并环丁烯基团对聚合物性能的 影响, 我们还利用双酚 $\mathrm{A}$ 单体合成了不带苯并环丁烯基 团的线性聚碳酸酯 PBAC, 以及将其与单体 BCB-DP、 三光气共聚获得部分侧链带有苯并环丁烯基团的共聚 物 BA-BPC. 其合成方法与 BPC 类似(Eq. 1), 并用相同 的表征手段对聚合物进行了表征。通过 GPC 测得 BA-BPC 和 BAPC 的数均分子量分别为 20500 和 19400 , 分散度均为 1.7 .

\section{3 聚碳酸酯的热固化行为}

鉴于苯并环丁烯结构在加热条件下发生的反应，聚 碳酸酯 BPC 固化后可以形成交联网状结构 PBPC, 其固 化反应示意图如 Scheme 2 所示. 苯并环丁烯中的四元 环可以开环聚合，也可以两两形成八元环，使得聚碳酸 酯主链间相互交联形成网状结构. 聚碳酸酯 BPC 的固 


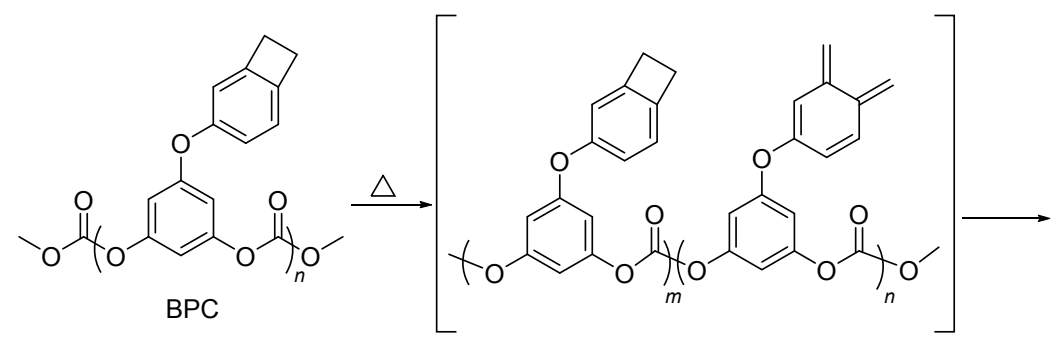

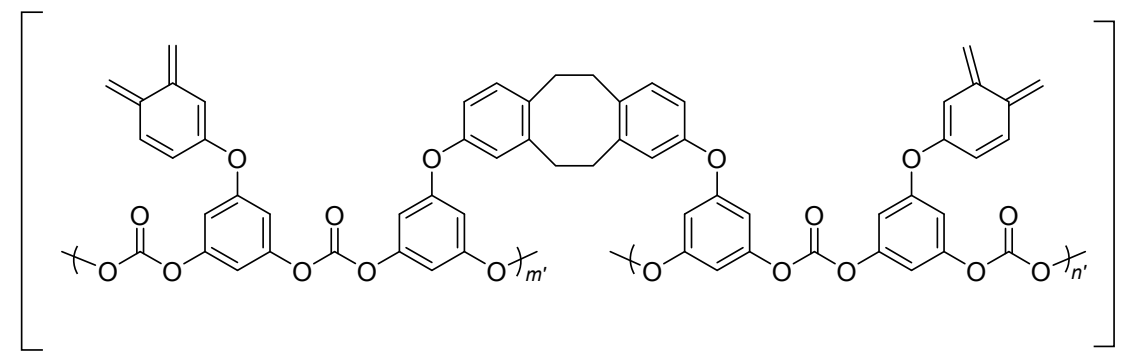

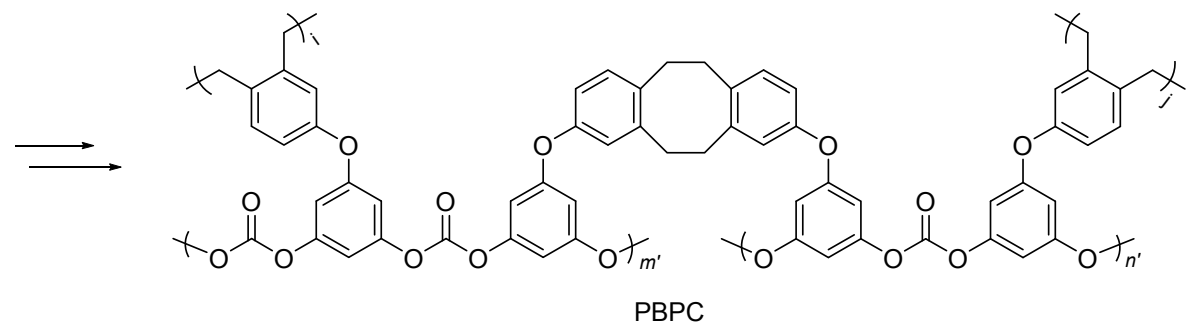

图式 2 聚碳酸酯 BPC 的固化反应示意图

Scheme 2 Curing reaction of BPC at high temperature

化行为可以用差扫描量热法(DSC)进行考察. 图 6 为 $\mathrm{BPC}$ 固化前后的 DSC 曲线. 图中 $93{ }^{\circ} \mathrm{C}$ 处的吸热峰为 $\mathrm{BPC}$ 的软化点, 聚合物的起始固化温度为 $197{ }^{\circ} \mathrm{C}$, 并且 其固化放热焓为 $248 \mathrm{~J} / \mathrm{g}$, 固化放热峰宽将近 $100{ }^{\circ} \mathrm{C}$, 表 明该聚碳酸酯具有较宽的加工窗口, 便于加工. 在第二 次升温扫描过程中 PBPC 在 $50 \sim 300{ }^{\circ} \mathrm{C}$ 范围内没有出 现明显的玻璃化转变, 表明其具有良好的耐热性. PBPC 的固化程度可通过红外光谱考察, 图 5 列出了 $\mathrm{BPC}$ 固化 前后的红外谱图, 可见 BPC 固化后, 苯并环丁烯基团在

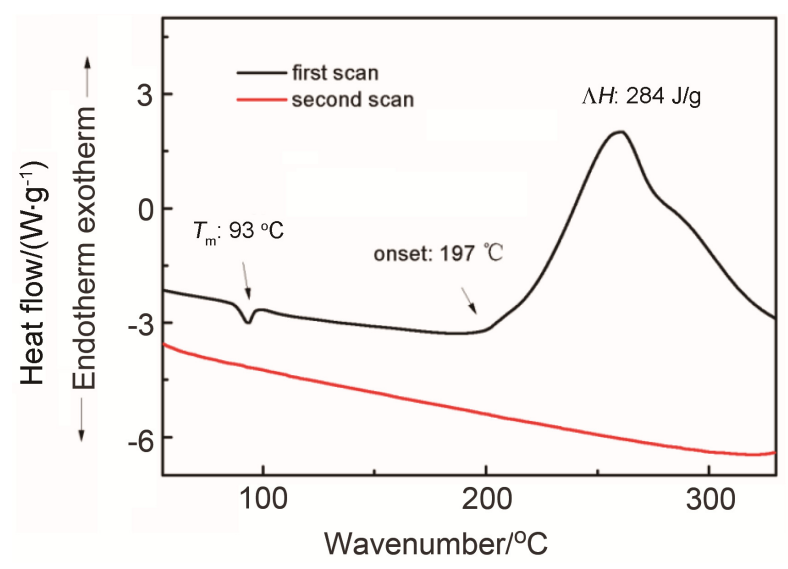

图 6 聚碳酸酯 BPC 固化前后的 DSC 曲线

Figure 6 DSC traces of BPC before and after cured
$1468 \mathrm{~cm}^{-1}$ 处的特征吸收峰消失，证明在该条件下聚合 物已固化完全形成交联网状的聚碳酸酯 PBPC. BA-BPC 在加热条件下有相似的固化行为，由于其苯并环丁烯基 团的含量较 BPC 少, 其交联产物 PBA-BPC 交联密度较 PBPC 低.

\section{4 固化后聚碳酸酯的热稳定性和疏水性}

为了研究苯并环丁烯基团对聚合物性能的影响, 我 们对 PBPC, PBA-BPC 以及不能固化的聚碳酸酯 BAPC 的热稳定性及疏水性进行了考察. 首先利用热重分析 (TGA)对三者的热稳定性进行了研究, 结果如表 1 所示. 在氮气氛围中, PBPC 的 $5 \%$ 热重损失温度高达 $451{ }^{\circ} \mathrm{C}$, $10 \%$ 的热重损失温度为 $473{ }^{\circ} \mathrm{C}, 1000{ }^{\circ} \mathrm{C}$ 下的残炭率为 $33 \%$, 展现了优异的热稳定性. PBA-BPC 的 5\%和 10\%的 热重损失温度分别 439 和 $454{ }^{\circ} \mathrm{C}$, 在 $1000{ }^{\circ} \mathrm{C}$ 下的残炭 率为 $23 \%$; 双酚 A 型聚碳酸酯 BAPC 的 5\%和 $10 \%$ 的热 重损失温度分别 412 和 $422{ }^{\circ} \mathrm{C}$, 其在 $1000{ }^{\circ} \mathrm{C}$ 下的残炭 率为 $19 \%$. 由此可见, 这三种聚合物中随着苯并环丁烯 基团含量的升高, 热稳定性逐渐提高. 这是由于苯并环 丁烯加热固化能形成交联网状结构，使得树脂具有高的 热稳定性. 因此, 在将苯并环丁烯引入聚碳酸酯中并通 过热固化交联后，使其热稳定性较线性双酚 $\mathrm{A}$ 型碳酸酯 有明显的提升. 
表 1 聚碳酸酯的热稳定性

Table 1 Thermostability of polycarbonates

\begin{tabular}{cccc}
\hline 聚碳酸酯 & $T_{5}{ }^{a}{ }^{\circ} \mathrm{C}$ & $T_{10}{ }^{a} /{ }^{\circ} \mathrm{C}$ & 残炭率 ${ }^{b} / \%$ \\
\hline BAPC & 412 & 422 & 19 \\
PBA-BPC & 439 & 454 & 23 \\
PBPC & 451 & 473 & 33 \\
\hline
\end{tabular}

${ }^{a} T_{5}$ 和 $T_{10}$ 分别代表在氮气氛围中质量损失为 $5 \%$ 和 $10 \%$ 时的温度; ${ }^{b}$ 氮气氛 围中 $1000{ }^{\circ} \mathrm{C}$ 下的残炭率

本文通过测试聚合物的吸水率, 及其与去离子水的 接触角分别对 PBPC, PBA-BPC 和 BAPC 的疏水性能进 行了比较研究. 结果如表 2 所示. 去离子水在 PBPC 薄 膜上的平均接触角为 $97^{\circ}$ (如图 7 所示), PBA-BPC 和 $\mathrm{BAPC}$ 的疏水角分别为 $90^{\circ}$ 和 $85^{\circ}$, 这表明随着苯并环丁 烯基团含量的增加, 聚合物表面疏水性逐步提高. 本文 对三种聚碳酸酯的吸水率进行了测试. 将三种聚碳酸酯 分别制备成 $5 \mathrm{~cm} \times 2.5 \mathrm{~cm}$ 的薄膜并放在沸水中浸泡 24 $\mathrm{h}$ 后, 计算浸泡前后薄膜的质量差, 其质量差与薄膜质 量的比值即为材料的吸水率. BAPC 的吸水率为 $0.39 \%$, 而含有苯并环丁烯的 PBA-BPC 和 PBPC 的吸水率分别 为 $0.28 \%$ 和 $0.21 \%$, 由此可见, PBPC 具有最好的疏水性. 这是因为苯并环丁烯为疏水性基团, 其热固化后, 使得 聚碳酸酯的交联密度变大, 疏水性的芳香基团和烷基的 比例增加, 从而使该聚碳酸酯疏水性有了明显的提高.

表 2 聚碳酸酯的吸水率及接触角测试

Table 2 Water uptake of polycarbonates

\begin{tabular}{cccc}
\hline 聚碳酸酯 & PBAC & PBA-BPC & PBPC \\
\hline 吸水率 $/ \%$ & $0.39 \%$ & $0.28 \%$ & $0.21 \%$ \\
接触角 $\left./{ }^{\circ}\right)$ & 85 & 90 & 97 \\
\hline
\end{tabular}

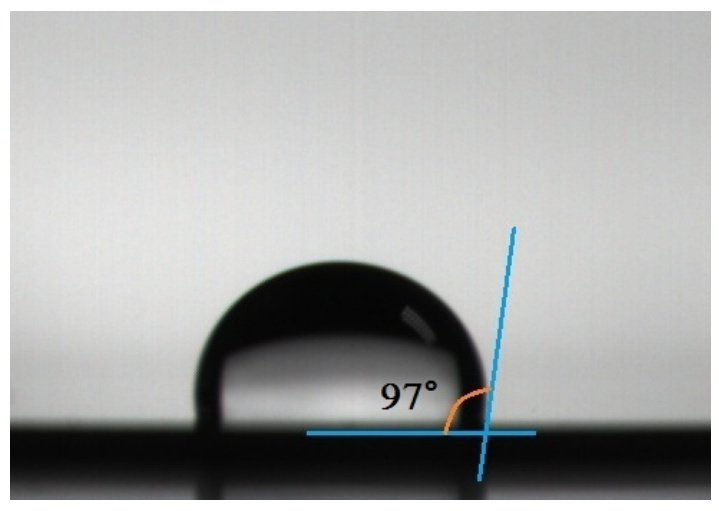

图 7 PBPC 薄膜表面与水接触角

Figure7 Contact angle of water on a PBPC film

\section{2 结论}

成功合成了一种新型的带有苯并环丁烯基团的双 酚单体 BCB-DP, 并以此发展了可热固化的聚碳酸酯 BPC. 该聚合物可用溶液法制备成薄膜, 加工性能良好. 在加热条件下发生固化, 得到具有交联网状结构的聚碳
酸酯 PBPC. 通过研究其性能可知, 该聚合物展现了优 异的耐热性和疏水性. 将其与部分侧链带有苯并环丁烯 基团的聚碳酸酯 BA-BPC 及线性双酚 $\mathrm{A}$ 聚碳酸酯 BAPC 的性能对比后可知，由于苯并环丁烯基团的引入，使得 聚碳酸酯的热稳定性及疏水性明显提高.

\section{3 实验部分}

\section{1 实验原料}

均三甲氧基苯(98\%)，玛雅试剂；正十二硫醇( $98 \%$ ), 阿达玛斯; 4-溴苯并环丁烯; 三光气( $99 \%)$, 双酚 A(99\%) 阿拉丁; 碘化亚铜, 铝, 阿法埃莎; 所用试剂均从上海 凌峰化学试剂有限公司或国药集团化学试剂有限公司 购买. 化合物 $\mathbf{1}$ 按已报道的方法合成 ${ }^{[23]}$, m.p. $37{ }^{\circ} \mathrm{C}$ (文 献值 ${ }^{[24]}$ m.p. $36 \sim 37{ }^{\circ} \mathrm{C}$ ).

\section{2 测试与表征仪器}

核磁氢谱采用 Bruker AV-400 核磁共振仪; 傅里叶 红外光谱(FT-IR)采用 Thermo Nicolet 380 红外光谱仪, KBr 压片法, 扫描范围 500 3500 $\mathrm{cm}^{-1}$; 示差扫描量热 法(DSC)采用 TA Q200 示差扫描量热仪, 氮气气流为 50 $\mathrm{mL} / \mathrm{min}$, 以 $10{ }^{\circ} \mathrm{C} / \mathrm{min}$ 的升温速率从 $40{ }^{\circ} \mathrm{C}$ 升温至 $350{ }^{\circ} \mathrm{C}$; 热重损失(TGA)采用 Netzsch TG $209 \mathrm{~F} 1$ 热重损 失分析仪, 以 $10{ }^{\circ} \mathrm{C} / \mathrm{min}$ 的升温速率从 $40{ }^{\circ} \mathrm{C}$ 升至 $1000{ }^{\circ} \mathrm{C}$; 接触角测试采用接触角测量仪 (JC2000C), 去 离子水作为测试液体; 分子量测定采用凝胶色谱仪 (GPC) Breeze2a 200, 四氢呋喃作为洗脱剂, 聚苯乙烯 作为标准对照样品.

\section{3 单体的合成}

\subsection{1 化合物 $\mathbf{2}$ 的合成}

在 $500 \mathrm{~mL}$ 烧瓶中, 加入化合物 1 (20.0 g, 129.7 $\mathrm{mmol}) 、$ 氢氧化钾 $(11.0 \mathrm{~g}, 194.6 \mathrm{mmol}) 、$ 甲苯 $(60 \mathrm{~mL})$ 和 $\mathrm{N}, \mathrm{N}$-二甲基乙酰胺 $(250 \mathrm{~mL})$, 在 $140{ }^{\circ} \mathrm{C}$ 下回流除水 $5 \mathrm{~h}$ 后，除去多余的甲苯，并冷却至室温. 加入溴代苯并环 丁烯 $(26.0 \mathrm{~g}, 142.7 \mathrm{mmol})$ 和碘化亚铜 $(2.5 \mathrm{~g}, 12.9 \mathrm{mmol})$, 冷冻抽换气三次后, 加热至 $140{ }^{\circ} \mathrm{C}$ 反应 $20 \mathrm{~h}$. 将反应液 冷却至室温, 倒入 $500 \mathrm{~mL}$ 冷水中, 并用 $100 \mathrm{~mL}$ 乙酸乙 酯萃取三次. 有机相用无水硫酸钠干燥, 除去溶剂后柱 层析分离, 得 $25.0 \mathrm{~g}$ 黄色油状物 2, 产率 75\%. ${ }^{1} \mathrm{H} \mathrm{NMR}$ $\left(400 \mathrm{MHz}, \mathrm{CDCl}_{3}\right) \delta: 6.99$ (d, $\left.J=7.9 \mathrm{~Hz}, 1 \mathrm{H}\right), 6.91 \sim 6.83$ $(\mathrm{m}, 1 \mathrm{H}), 6.76(\mathrm{~d}, J=1.5 \mathrm{~Hz}, 1 \mathrm{H}), 6.20 \sim 6.09(\mathrm{~m}, 3 \mathrm{H})$, $3.73(\mathrm{~s}, 6 \mathrm{H}), 3.13(\mathrm{~s}, 4 \mathrm{H})$. HRMS calcd for $\mathrm{C}_{16} \mathrm{H}_{16} \mathrm{O}_{3}$ 256.1099 , found 256.1104 .

\subsection{2 化合物 BCB-DP 的合成}

在 $500 \mathrm{~mL}$ 烧瓶中, 加入铝(13.2 g, $487.7 \mathrm{mmol})$ 和乙 腈 $(250 \mathrm{~mL})$, 之后慢慢加入碘 $(130 \mathrm{~g}, 487.7 \mathrm{mmol})$. 加热 
至 $85{ }^{\circ} \mathrm{C}$ 回流反应 $3 \mathrm{~h}$, 冷却至室温后, 加入化合物 2 $(24.8 \mathrm{~g}, 96.8 \mathrm{mmol})$, 再加热至 $85{ }^{\circ} \mathrm{C}$ 反应 $5 \mathrm{~h}$. 反应物冷 却至室温后, 倒入至稀盐酸的冷水溶液中, 用 $100 \mathrm{~mL}$ 乙酸乙酯萃取三次. 有机相用无水硫酸钠干燥, 除去溶 剂后柱层析分离, 得 $21.0 \mathrm{~g}$ 白色固体 BCB-DP, 产率 95\%. m.p. 30 32 ${ }^{\circ} \mathrm{C}$; ${ }^{1} \mathrm{H}$ NMR (400 MHz, $\left.\mathrm{CDCl}_{3}\right) \delta$ : $7.01(\mathrm{~d}, J=7.8 \mathrm{~Hz}, 1 \mathrm{H}), 6.87(\mathrm{~d}, J=7.9 \mathrm{~Hz}, 1 \mathrm{H}), 6.77$ (s, $1 \mathrm{H}), 6.02$ (dt, $J=9.3,2.0 \mathrm{~Hz}, 3 \mathrm{H}), 5.07$ (s, 2H), 3.14 (d, $J=1.8 \mathrm{~Hz}, 4 \mathrm{H})$; HRMS calcd for $\mathrm{C}_{14} \mathrm{H}_{12} \mathrm{O}_{3} 228.0786$, found 228.0787 .

\subsection{3 聚碳酸酯的合成}

含苯并环丁烯基团聚碳酸酯(BPC)的合成: 将 100 $\mathrm{mL}$ 两口瓶干燥, 氮气保护下, 分别加入化合物 BCB-DP $(1.00 \mathrm{~g}, 4.38 \mathrm{mmol})$ 和三光气 $(1.30 \mathrm{~g}, 4.38 \mathrm{mmol})$, 溶解于 $30 \mathrm{~mL}$ 氯仿. 在冰水浴条件下, 缓慢滴加三乙胺 $(1.30$ $\mathrm{mL}, 8.76 \mathrm{mmol})$ 的氯仿溶液, 滴加完毕后, 撤除冰水浴, 在室温下反应 $5 \mathrm{~h}$, 加入过量无水甲醇封端. 反应完毕 后, 倒入 $50 \mathrm{~mL}$ 水中, 用氯仿 $(30 \mathrm{~mL})$ 萃取三次, 有机相 用无水硫酸钠干燥后浓缩, 于无水甲醇中沉析, 将沉淀 过滤, 真空干燥后得到白色固体 $0.86 \mathrm{~g}$, 产率 77\%. m.p. $93{ }^{\circ} \mathrm{C}$; ${ }^{1} \mathrm{H}$ NMR $\left(400 \mathrm{MHz}, \mathrm{CDCl}_{3}\right) \delta: 6.64 \sim 7.12(\mathrm{~m}$, $6 \mathrm{H}), 3.13$ (s, 4H); FT-IR (KBr) v: 2925, 1786, 1625, 1601, $1468,1215,1111,992 \mathrm{~cm}^{-1}$. GPC: $M_{\mathrm{n}}=25000, M_{\mathrm{w}} / M_{\mathrm{n}}=$ 1.6 .

共聚聚碳酸酯(BA-BPC)的合成: 将 $100 \mathrm{~mL}$ 两口瓶 干燥, 氮气保护下, 分别加入化合物 3 (1.00 g, 4.38 $\mathrm{mmol})$ 、双酚 $\mathrm{A}(1.00 \mathrm{~g}, 4.38 \mathrm{mmol})$ 和三光气(2.60 g, 8.76 $\mathrm{mmol})$, 溶解于 $60 \mathrm{~mL}$ 氯仿. 在冰水浴冷却下, 缓慢滴加 三乙胺 $(2.6 \mathrm{~mL}, 17.52 \mathrm{mmol})$ 的氯仿溶液, 滴加完毕后, 撤除冰水浴, 在室温下反应 $5 \mathrm{~h}$, 加入过量无水甲醇封 端. 反应完毕后, 倒入 $100 \mathrm{~mL}$ 水中, 用氯仿 $(60 \mathrm{~mL})$ 萃 取三次, 有机相用无水硫酸钠干燥后浓缩, 于无水甲醇 中沉析, 将沉淀过滤后真空条件下干燥, 得到白色固体 $1.56 \mathrm{~g}$, 产率 84\%. m.p. $113{ }^{\circ} \mathrm{C}$; ${ }^{1} \mathrm{H}$ NMR (400 MHz, $\left.\mathrm{CDCl}_{3}\right) \delta: 6.74 \sim 7.23(\mathrm{~m}, 16 \mathrm{H}), 3.14(\mathrm{~s}, 4 \mathrm{H}), 1.67(\mathrm{~s}, 9 \mathrm{H})$; FT-IR (KBr) $v: 2973,1783,1622,1601,1468,1200,1018$, $837 \mathrm{~cm}^{-1}$. GPC: $M_{\mathrm{n}}=20500, M_{\mathrm{w}} / M_{\mathrm{n}}=1.7$.

线性双酚 $\mathrm{A}$ 型聚碳酸酯(BAPC)的合成: 将 $100 \mathrm{~mL}$ 两口瓶干燥, 氮气保护下, 分别加入双酚 A (1.00 g, 4.38 $\mathrm{mmol})$ 和三光气 $(1.30 \mathrm{~g}, 4.38 \mathrm{mmol})$, 并溶于 $30 \mathrm{~mL}$ 氯仿 中. 在冰水浴条件下, 缓慢滴加三乙胺 $(1.3 \mathrm{~mL}, 8.76$ $\mathrm{mmol})$ 的氯仿溶液, 滴加完毕后, 撤除冰水浴, 在室温 下反应 $5 \mathrm{~h}$, 加入过量无水甲醇封端. 反应完毕后, 倒入 $50 \mathrm{~mL}$ 水中, 用氯仿 $(30 \mathrm{~mL})$ 萃取三次, 有机相用无水硫 酸钠干燥后浓缩, 于无水甲醇中沉析, 将沉淀过滤后真
空条件下干燥, 得到白色固体 $0.95 \mathrm{~g}$, 产率 $85 \%$. ${ }^{1} \mathrm{H}$ NMR (400 MHz, $\left.\mathrm{CDCl}_{3}\right) \delta: 7.13 \sim 7.24(\mathrm{~m}, 8 \mathrm{H}), 1.66(\mathrm{~s}$, $6 \mathrm{H})$; FT-IR (KBr) v: 2970, 1771, 1715, 1604, 1506, 1230, $1120,1164,1015,831 \mathrm{~cm}^{-1}$. GPC: $M_{\mathrm{n}}=19400, M_{\mathrm{w}} / M_{\mathrm{n}}=$ 1.6 .

\section{4 聚碳酸酯薄膜的制备、固化及性能测试}

将聚碳酸酯溶于 1,2 -二氯乙烷中, 浓度为 200 $\mathrm{mg} / \mathrm{mL}$, 待白色固体全部溶解, 滴涂在 $2.5 \mathrm{~cm} \times 2.5 \mathrm{~cm}$ 玻璃片上, 待溶剂自然挥发干后转移至真空干燥箱中保 持在 $85{ }^{\circ} \mathrm{C}$, 使溶剂充分挥发完全, 得到固体薄膜. 将 除去溶剂的薄膜样品在真空管式炉中梯度升温固化, 梯 度升温过程为: $150{ }^{\circ} \mathrm{C} / 1 \mathrm{~h}, 200{ }^{\circ} \mathrm{C} / 1 \mathrm{~h}, 250{ }^{\circ} \mathrm{C} / 3 \mathrm{~h}$, $300{ }^{\circ} \mathrm{C} / 3 \mathrm{~h}$. 样品自然冷却至室温后得到固化后的聚合 物薄膜.

按以上方法制得 $5 \mathrm{~cm} \times 2.5 \mathrm{~cm}$ 固化后的聚碳酸酯 薄膜, 在真空烘箱中干燥至恒重后再在干燥器中自然冷 却至室温, 将样品置于 $100 \mathrm{~mL}$ 去离子水中, 加热至沸 腾状态保持 $24 \mathrm{~h}$ 后, 将样品取出. 用滤纸迅速擦去薄膜 表面的水分，在室温下称重，计算吸水率.

辅助材料(Supporting Information) 化合物 1 和 $\mathbf{2}$ 的 ${ }^{1} \mathrm{H}$ NMR 谱. 单体 BCB-DP 及聚合物 BPC, BA-BPC, BAPC 的 ${ }^{1} \mathrm{H}$ NMR 谱和 FT-IR 谱图. 这些材料可以免费从本刊 网站(http://sioc-journal.cn/)上下载.

\section{References}

[1] Bendler, J. T.; Boyles, D. A.; Edmondson, C. A.; Filipova, T.; Fontanella, J. J.; Westgate, M. A.; Wintersgill, M. C. Macromolecules 2013, 46, 4024.

[2] Yoonessi, M.; Gaier, J. R. ACS Nano 2010, 4, 7211.

[3] Tamarit-López, J.; Morais, S.; Bañuls, M.; Puchades, R.; Maquieira, Á. Anal. Chem. 2010, 82, 1954.

[4] Zhou, J.; Lubineau, G. ACS Appl. Mater. Interfaces 2013, 5, 6189.

[5] Méndez-Liñán, L.; López-Garzón, F. J.; Domingo-García, M.; Pérez-Mendoza, M. Energy Fuels 2010, 24, 3394.

[6] Moon, S. I.; Extrand, C. W. Ind. Eng. Chem. Res. 2009, 48, 8961.

[7] Lyu, M.-Y.; Lee, J. S.; Pae, Y. J. Appl. Polym. Sci. 2001, 80, 1814.

[8] Baick, I. H.; Yang, W. J.; Ahn, Y. G.; Song, K. H.; Choi, K. Y. J. Appl. Polym. Sci. 2015, 132, 41609.

[9] Awwad, S. H.; Filipova, T. S.; Boyles, D. A. Polym. Prepr. 2011, 52 (1), 368 .

[10] Laughner, M. K.; Farah, H. WO 9215643, 1992 [Chem. Abdtr. 1993, 118, 256003].

[11] Boyles, D. A.; Filipova, T. S.; Bendler, J. T.; Longbrake, G.; Reams, J. Macromolecules 2005, 38, 3622.

[12] Filipova, T. S.; Boyles, D. A.; Schroeder, M. J.; Fontanella, J. J.; Wintersgill, M. C.; Edmondson, C. A.; Bendler, J. T. Polym. Prepr. 2009, 50(2), 543

[13] Delassus, S. L.; Howell, B. A. Macromolecules 1994, 27, 1307.

[14] Tian, S.; Sun, J.; Jin, K.; He, F.; Zheng, S.; Fang, Q. ACS Appl. Mater. Interfaces 2014, 6, 20437.

[15] Hahn, S. F.; Martin, S. J.; Mckelvy, M. L.; Patrick, D. W. Macro- 
molecules 1993, 26, 3870

[16] Marks, M. J.; Erskine, J. S.; McCrery, D. A. Macromolecules 1994, 27, 4114.

[17] Wang, J.; Piskun, I.; Craig, S. L. ACS Macro Lett. 2015, 4, 834.

[18] Cheng, Y.; Yang, J.; Jin, Y.; Deng, D.; Xiao, F. Macromolecules 2012, 45, 4085.

[19] He, F.; Yuan, C.; Li, K.; Diao, S.; Jin, K.; Wang, J.; Tong, J.; Ma, J.; Fang, Q. RSC Adv. 2013, 3, 23128.

[20] Ma, B.; Lauterwasser, F.; Deng, L.; Zonte, C. S.; Kim, B. J.; Fré- chet, J. M. J. Chem. Mater. 2007, 19, 4827.

[21] Walker, K. A.; Markoski, L. J.; Moore, J. S. Macromolecules 1993, 26, 3713.

[22] Wang, Y.; Sun, J.; Jin, K.; Wang, J.; Yuan, C.; Tong, J.; Diao, S.; He, F.; Fang, Q. RSC Adv. 2014, 4, 39884.

[23] Chae, J. Arch. Pharm. Res. 2008, 31, 305.

[24] Kampouris, E. M. J. Chem. Soc. 1965, 2651.

[25] Yang, J.; Liu, S.; Zhu, F.; Huang, Y.; Li, B.; Zhang, L. J. Polym. Sci., Part A: Polym. Chem. 2011, 49, 381. 